\title{
Hyperglycaemia in post-surgical patients receiving oral, enteral and parenteral nutrition: is insulin sliding scale therapy sufficient?
}

\author{
J. Bhullar, K. Chung and T. M. Trebble \\ Department of Gastroenterology, Queen Alexandra Hospital, Portsmouth, PO6 3LY, UK
}

\begin{abstract}
Hyperglycaemia is a common complication of the immediate post-operative state. It is associated with increased rates of sepsis, amongst other complications, and increased mortality. Poor glycaemic control may be seen in pre-existing diabetics and non-diabetics secondary to insulin resistance, and is actively managed with an intravenous insulin sliding scale (IVSS). Queen Alexandra Hospital is a large district general hospital with a high throughput of complex surgical presentations. A previous audit demonstrated hyperglycaemia in approximately $25 \%$ of patients post-surgery. We undertook a prospective clinical audit to assess the effectiveness of IVSS intervention in postoperative patients receiving oral, enteral and parenteral nutrition.

Pre-determined standards relating to frequency of monitoring and effectiveness of glycaemic control were audited. Data were collected from 23 patients (mean age 63.4 years, range 35-93 years) who had confirmed hyperglycaemia with a blood glucose $>8 \mathrm{mmol} / \mathrm{l}$ on at least two occasions, and were commenced on an IVSS. There were 14 patients with known type 2 diabetes mellitus (DM) and one patient with type $1 \mathrm{DM}$; eight patients had no history of diabetes. Seven patients received nutritional support as TPN (6), enteral nutrition (1) or oral supplements (5).

Blood glucose (BG) levels were monitored on average every 4.6 hours. There was no correlation between the level of nursing care and frequency of BG monitoring. Nineteen ( $82 \%$ ) patients receiving IVSS failed to reach the $80 \%$ target of maintaining BG levels between 4 and $8 \mathrm{mmol} / \mathrm{l}$. The mean percentage of BG readings above $8 \mathrm{mmol} / \mathrm{l}$ was $52 \%$. Three patients in particular were persistently hyperglycaemic with all BG levels above $8 \mathrm{mmol} / \mathrm{l}$. Eight patients had an associated infection or positive microbiology, which was proportionally more common in the TPN group, despite apparently more stable glycaemic control. Four (17\%) patients experienced documented hypoglycaemic events while on a sliding scale regimen. Most patients (21) received intravenous fluids as part of their sliding scale regime; of the 13 patients with a documented fluid balance, seven were in positive fluid balance.

Our results suggest that an intravenous insulin sliding scale may not provide effective glycaemic control in patients in the post-operative state, in terms of both hyper- and hypoglycaemia. Patients receiving TPN appeared to be more stable, possibly reflecting a more constant calorific intake. Further issues relate to the contribution of the IVSS to fluid overload in this patient group and the required demands of the IVSS on staff time. Research is indicated to examine alternative methods of glycaemic control in hyperglycaemic post-operative patients.
\end{abstract}

